ARTICLE OPEN

\title{
The influences of El Nino and Arctic sea-ice on the QBO disruption in February 2016
}

\author{
N. Hirota ${ }^{1}$, H. Shiogama (iD ${ }^{1}$, H. Akiyoshi ${ }^{1}$, T. Ogura ${ }^{1}$, M. Takahashi ${ }^{1,2}$, Y. Kawatani $^{3}$, M. Kimoto ${ }^{2}$ and M. Mori $^{4}$
}

The westerly phase of the quasi-biennial oscillation (QBO) was unexpectedly disrupted by an anomalous easterly near $40 \mathrm{hPa}$ $(\sim 23 \mathrm{~km})$ in February 2016. At the same time, a very strong El Nino and a very low Arctic sea-ice concentration in the Barents and Kara Sea were present. Previous studies have shown that the disruption of the QBO was primarily caused by the momentum transport of the atmospheric waves in the Northern Hemisphere. Our results indicate that the tropical waves evident over the Atlantic, Africa, and the western Pacific were associated with extratropical disturbances. Moreover, we suggest that the El Nino and sea-ice anomalies in 2016 account for approximately half of the disturbances and waves based on multiple regression analysis of the observational/reanalysis data and large-ensemble experiments using an atmospheric global climate model.

npj Climate and Atmospheric Science (2018)1:10 ; doi:10.1038/s41612-018-0020-1

\section{INTRODUCTION}

The quasi-biennial oscillation (QBO) is the dominant mode of stratospheric variability with a period of 22-36 months. ${ }^{1}$ The oscillation is characterized by alternating easterlies and westerlies descending from $\sim 1 \mathrm{hPa}(\sim 50 \mathrm{~km})$ to $\sim 100 \mathrm{hPa}(\sim 16 \mathrm{~km})$ and has been observed to occur in a very similar manner for several decades. ${ }^{2,3}$ Due to its strong periodicity and its various influences on the atmosphere, the $\mathrm{QBO}$ is an important factor in the predictability of the seasonal forecasts. ${ }^{4}$ However, in February 2016 the descent of the westerly phase of the QBO was unexpectedly disrupted by an easterly in the lower stratosphere near $40 \mathrm{hPa}$. As emphasized in previous studies, this disruption was completely unprecedented and not predicted by the forecast models., ${ }^{5,6}$ The primary cause of the easterly was the momentum transport from the planetary waves from the Northern Hemisphere converging near the equator. ${ }^{6,7}$ Previous studies also mentioned that the $\mathrm{El}$ Nino in 2016 was a leading candidate for the source of the tropospheric waves that caused the QBO disruption. 5,7,8 However, El Nino alone cannot explain this event as the QBO westerly in 1998 was not disrupted by the very strong El Nino occurring at that time. Additionally, we examine the recent reduction of the Arctic sea-ice, which is associated with significant atmospheric disturbances in the Northern Hemisphere., ${ }^{9,10}$ This study aims to understand why the unprecedented disruption occurred in 2016.

\section{RESULTS}

Figure 1 presents an overview of the unexpected disruption event of the QBO in 2016 and the associated atmospheric waves based on the Modern-Era Retrospective analysis for Research and Applications, Version 2 (MERRA2; see Methods for details). The waves are examined using the Eliassen-Palm (EP) flux ${ }^{11}$ and the threedimensional wave activity flux (WAF) ${ }^{12}$ which run parallel to the group velocity, identifying the propagation direction of the waves.
During 2015, the westerly phase of the QBO descended in the stratosphere. However, during 2016 the westerly descent at $20 \mathrm{~Pa}$ halted while an easterly developed near $40 \mathrm{hPa}$ (Fig. 1a). Correspondingly, the waves in the Northern Hemisphere propagated from the extratropical troposphere to the equatorial stratosphere (Fig. 1b). The associated wave divergence was negative, i.e., the easterlies accelerate, ${ }^{11}$ in the equatorial lower stratosphere (Fig. 1a). The horizontal wave propagation as indicated by the WAF shows that the equatorward traveling waves were apparent over the Atlantic, Africa, and the western Pacific (Fig. 1c). We emphasize that the tropical waves shown by the EP flux in the previous studies ${ }^{6,7}$ were zonally localized. Note that the vectors above $100 \mathrm{hPa}$ (Fig. $1 \mathrm{~b}$ ) and equatorward of $30^{\circ} \mathrm{N}$ (Fig. 1b, c) are multiplied by factors of 5 and 10, respectively.

Figure $1 \mathrm{~d}$ compares the magnitudes of the EP flux divergences in February of the past 37 (1980-2016) years with the QBO zonal winds of the equatorial lower stratosphere. These values are normalized by the standard deviations of their year-to-year variations. The EP flux divergence in 2016 shows a very large negative value of -2.6 in the QBO westerly (zonal wind of 0.25 ). The large negative value in 2016 is also evident in the time series of the EP flux divergence shown in Fig. S1. The EP flux divergence in 2010 also shows large negative values. However, in the case of 2010, the zonal wind was easterly, and the QBO disruption did not occur, unlike in 2016. Stationary Rossby waves cannot propagate when the mean zonal winds are easterly.

The stream function anomaly in the extratropics had the horizontal structure of zonal wavenumbers 1 and 2 (Fig. 1c), which corresponded to the significant meandering of the westerly jet with the disturbed potential vorticity (PV) field in February (Fig. 2). Examining the daily data from February, we see that the wave structure in the extratropics was basically steady, whereas the amplitude of each anomaly varied with time. Figure $2 b$, c show two different days: On February 6th, the positive anomaly of the

\footnotetext{
${ }^{1}$ Center for Global Environmental Research, National Institute for Environmental Studies, Tsukuba, Japan; ${ }^{2}$ Atmosphere and Ocean Research Institute, The University of Tokyo,

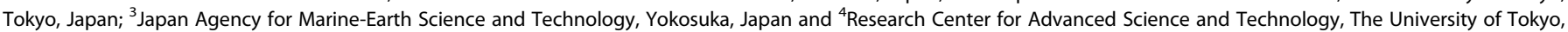
Tokyo, Japan

Correspondence: N. Hirota (nagioh@gmail.com)
}

Received: 7 September 2017 Revised: 12 January 2018 Accepted: 12 March 2018

Published online: 25 May 2018 
(a) [MERRA2] U(shd) \& EPdiv(cnt) $(10 S-10 N)$

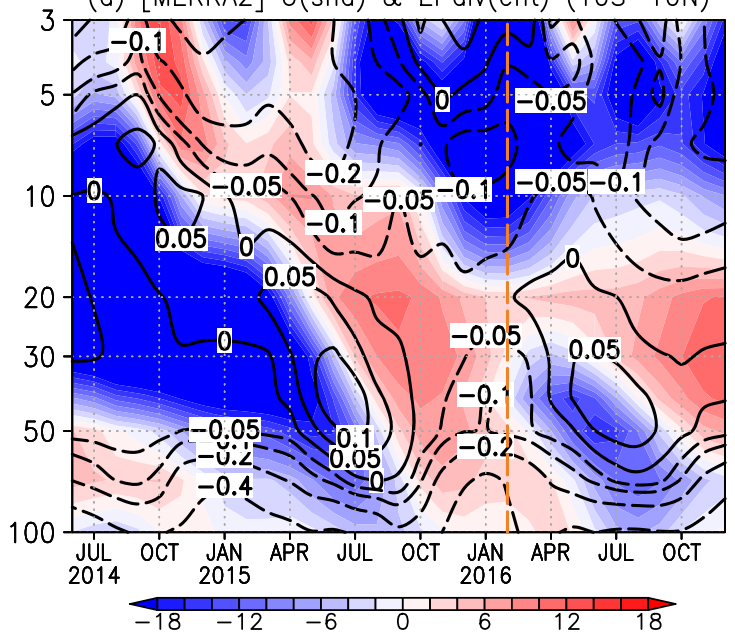

(c) [MERRA2] psi(shd) \& WAF(vct) $40 \mathrm{hPa} \mathrm{Feb}$

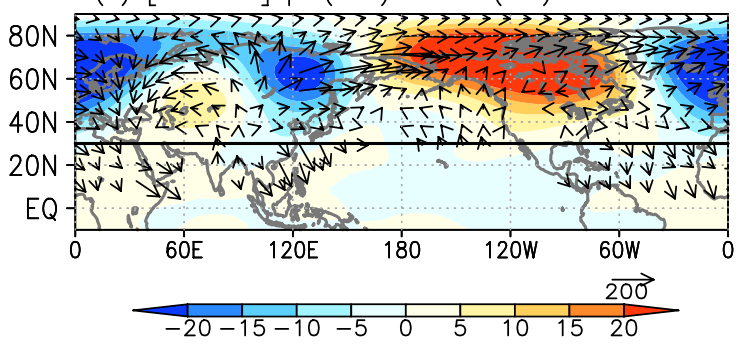

(b) [MERRA2] U(shd) \& EP(vct) Feb

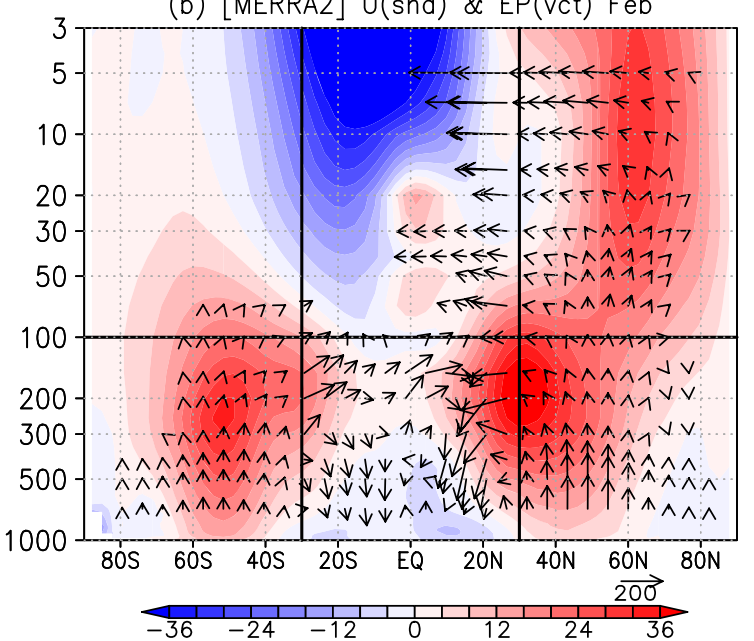

(d) [MERRA2] Feb1980-2016

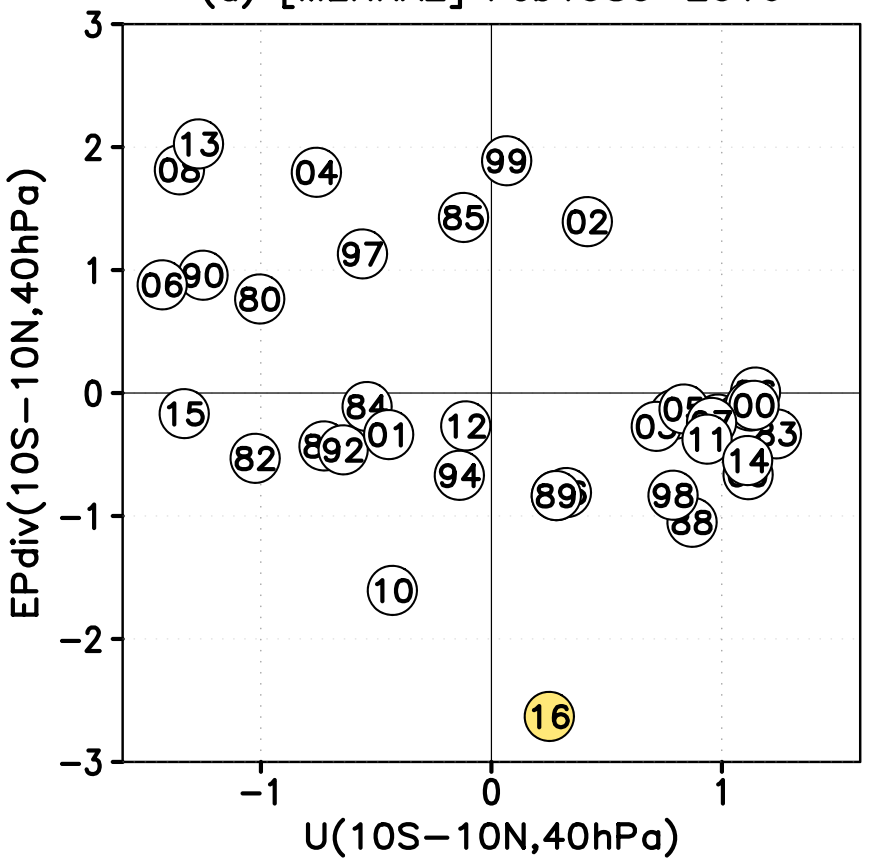

Fig. 1 An overview of the QBO disruption in 2016. a The time evolution of the zonal mean zonal wind (shading; $\mathrm{m} \mathrm{s}^{-1}$ ) and the EP flux divergence (contour; $0, \pm 0.05, \pm 0.1, \pm 0.2, \pm 0.4 \mathrm{~m} \mathrm{~s}^{-1}$ day $^{-1}$ ) near the equator $\left(10^{\circ} \mathrm{S}-10^{\circ} \mathrm{N}\right)$. The orange dashed line indicates February 2016 . $\mathbf{b}$ The zonal mean zonal wind (shading; $\mathrm{m} \mathrm{s}^{-1}$ ) and the EP flux (vector) of February 2016. (c) The anomalies of the stream function (shading; $10^{6}$ $\mathrm{m}^{2} \mathrm{~s}$; zonal mean is removed) and WAF (vector; $\mathrm{m}^{2} \mathrm{~s}^{2}$ ) at $40 \mathrm{hPa}$. The vectors above $100 \mathrm{hPa}$ in (b) and equatorward of $30^{\circ} \mathrm{N}$ in $(\mathbf{b}, \mathbf{c})$ are multiplied by factors of 5 and 10, respectively. d A scatter plot of the EP flux divergence and the zonal wind averaged over the equatorial lower stratosphere $\left(10^{\circ} \mathrm{S}-10^{\circ} \mathrm{N}, 40 \mathrm{hPa}\right)$ for February $1980-2016$ (values are normalized by their respective standard deviations)

stream function over North America was enhanced, with the larger equatorward WAF over the tropical Atlantic and Africa. On February 23rd, the WAF over the Atlantic and Africa was relatively small, while the stream function anomaly over eastern Eurasia and the equatorward WAF over the tropical western Pacific were stronger. Moreover, comparing the time series of the EP flux divergence associated with disturbances of all wavenumbers and those of wavenumbers 1 and 2 (black solid and dashed lines in Fig. S1), we see that its variabilities were dominated by the wavenumbers 1 and 2 components (the temporal correlation is $0.84)$. These results illustrate that the equatorward waves in the lower stratosphere were associated with the quasi-stationary disturbances of the atmospheric circulations in the extratropics.

Next, we explore the factors that contribute to the formation of the anomalous QBO by examining the impacts of the strong El
Nino and sea-ice anomaly in February 2016. We use the NINO3.4 index (sea surface temperature (SST) over the equatorial eastern Pacific $\left(170-120^{\circ} \mathrm{W}, 5^{\circ} \mathrm{S}-5^{\circ} \mathrm{N}\right)$; a large positive value indicates a strong El Nino) to measure the strength of the El Nino, and define a sea-ice index (SIC) by averaging the Arctic sea-ice concentrations in the Barents and Kara Seas $\left(30-90^{\circ} \mathrm{E}, 65-85^{\circ} \mathrm{N}\right)$, where the sea-ice anomaly in 2016 was large (Fig. S1 and Fig. S2). The observational dataset of the SSTs and sea-ice concentrations was compiled by the Hadley Center.

Figure 3a plots the normalized NINO3.4 and the SIC for the past 37 years. The value of NINO3.4 in February 2016 is 1.7, which is the third strongest El Nino in the analyzed period, whereas the sea-ice concentration is the smallest, with a value of -2.0 (SIC). Although some years have a strong El Nino (1983 and 1998) or low sea-ice concentrations (2008 and 2012), 2016 clearly stands out as having 
(a)[MERRA2] pv WAF psi 40hPa FEB

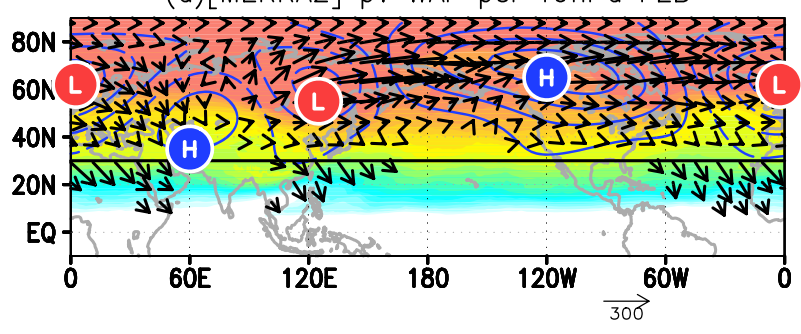

(b)[MERRA2] pv WAF psi $40 \mathrm{hPa}$ 06FEB

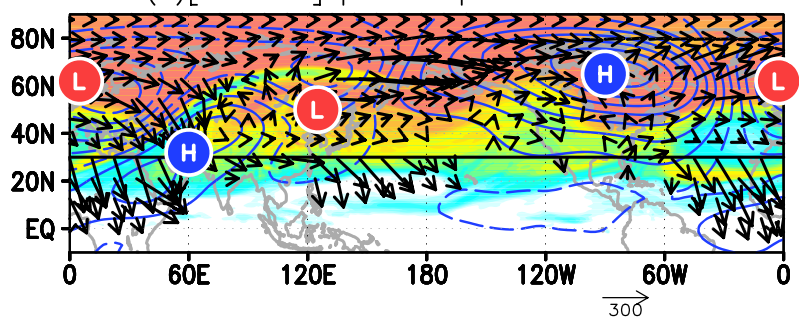

(c)[MERRA2] pv WAF psi 40hPa 23FEB

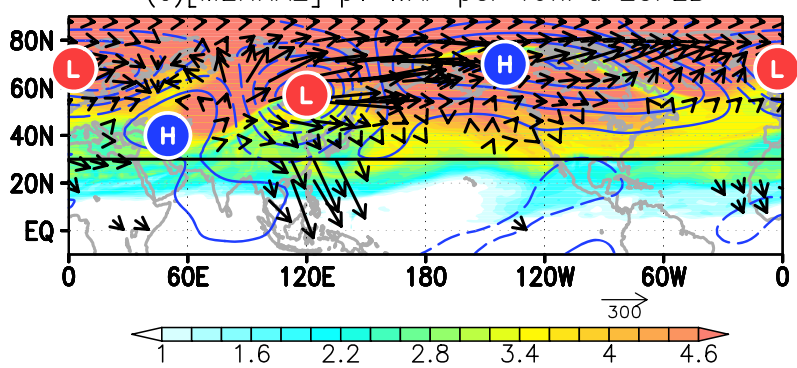

Fig. 2 Atmospheric disturbances and waves in the lower stratosphere. PV (shading; $10^{-5} \mathrm{~K} \mathrm{~kg}^{-1} \mathrm{~m}^{2} \mathrm{~s}^{-1}$ ), WAF (vector; $\mathrm{m}^{2} \mathrm{~s}^{-2}$ ) and anomalous stream function (contour; $10^{6} \mathrm{~m}^{2} \mathrm{~s}^{-1}$; zonal mean is removed) at $40 \mathrm{hPa}$ for (a) the February average, (b) on February 6th, and (c) on February 23rd. The vectors in the region equatorward of $30^{\circ} \mathrm{N}$ are multiplied by a factor of $10 . \mathrm{H}$ and $\mathrm{L}$ indicate anticyclonic and cyclonic anomalies, respectively

both a strong El Nino and a low sea-ice concentration. The correlation between NINO3.4 and SIC is small (0.07), suggesting that they are independent of each other.

The impacts of El Nino and sea-ice are further investigated using a multiple regression analysis and the least square method. Circulation anomalies are expressed as $\mathrm{X}^{\prime}=a \mathrm{NINO} .4+\beta \mathrm{SIC}+\gamma$, where $a, \beta, \gamma$ are the regression coefficients. Figure $3 \mathrm{~b}$ shows the regression map of $a \mathrm{NINO3.4}+\beta \mathrm{SIC}$, with values of 1.7 for NINO3.4 and -2.0 for SIC in 2016. The regression map of the stream function is similar to the anomaly pattern in 2016 (Fig. 1c; the pattern correlation is 0.83). Moreover, the WAF in the tropics is evident over the Atlantic, Africa and the western Pacific, as is seen in the anomalies of 2016. Meanwhile, the amplitude of the regression is approximately half of the 2016 anomaly pattern (the color scale and the lengths of the vectors in Fig. $3 b$ are half those in Fig. 1c). We suggest that El Nino and the Arctic sea-ice are important because they account for approximately half of the circulation anomalies and the associated waves in the lower stratosphere. The inter-annual variabilities of the equatorward waves are also partially related with El Nino and sea-ice. The temporal correlation between the EP flux divergence (a black line in Fig. S1) and that predicated by the multiple regression $(a \mathrm{NINO3.4}(\mathrm{t})+\beta \mathrm{SIC}(\mathrm{t})$; a black dotted line in Fig. $\mathrm{S} 1)$ is 0.46 .

To further investigate the impacts of El Nino and the large retreat of sea-ice on the lower stratospheric circulations, we
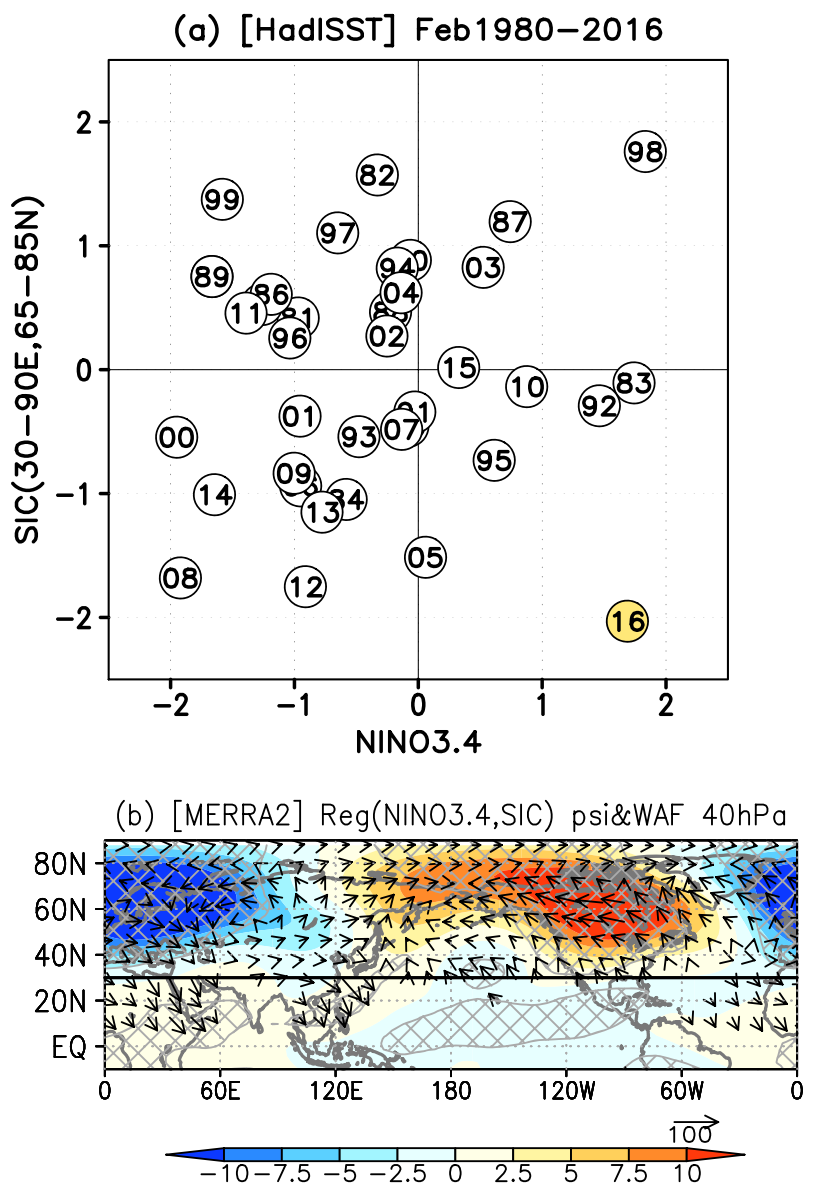

Fig. $3 \mathrm{El}$ Nino and Arctic sea-ice in relation to the lower stratospheric circulations. a A scatter plot of NINO3.4 and the seaice over the Barents and Kara Sea $\left(30-90^{\circ} \mathrm{E}, 65-85^{\circ} \mathrm{N}\right)$ for February 1980-2016 (values are normalized). b A multiple regression map of the stream function (shading; $10^{6} \mathrm{~m}^{2} \mathrm{~s}^{-1}$; zonal mean is removed) and WAF (vector; $\mathrm{m}^{2} \mathrm{~s}^{-2}$ ) at $40 \mathrm{hPa}$ with respect to NINO3.4 and the sea-ice. The hatchings denote a significance level of $90 \%$ based on the bootstrap method. The regression coefficients are weighted by the respective values of NINO3.4 (1.7) and the sea-ice $(-2.0)$ in February 2016 for a comparison with the anomaly of February 2016. The vectors equatorward of $30^{\circ} \mathrm{N}$ are multiplied by a factor of 10

examine the output data from the large-ensemble experiments of the atmospheric global climate model (AGCM; see Methods for the model details) as part of the attribution experiments for extreme events. ${ }^{13}$ We analyze three 100-member ensemble experiments with different initial conditions: 1) ALL: Control simulations of 2016 with boundary conditions of the historical SST, sea-ice, and radiative forcing agents; 2) noENSO: Same as ALL but the SST anomaly regressed onto Nino3.4 was removed; 3) dtrSIC: Same as ALL but the long-term 1870-2016 trend of the decreasing sea-ice was removed. ${ }^{14}$ In addition, we analyze the control experiment of 1980-2016 (ALL-LNG; 10-member ensemble) to evaluate the model climatology (see Methods).

Figure $4 a, b$ show the ensemble averaged stream functions of ALL minus noENSO and ALL minus dtrSIC, showing the impacts of the strong El Nino and the low sea-ice concentration, respectively, in 2016. Each response shows a negative value over the North Atlantic to Europe and a positive value over North America, resembling the circulation anomaly of 2016 (Fig. 1c; the pattern correlations with Fig. $4 a, b$ are 0.59 and 0.63 , respectively). We define the amplitudes of the extratropical disturbances using the maximum value of the stream function anomaly over $\left(150^{\circ} \mathrm{E}-60^{\circ}\right.$ $\left.\mathrm{W}, 50-80^{\circ} \mathrm{N}\right)$ minus the minimum value over $\left(60^{\circ} \mathrm{W}-150^{\circ} \mathrm{E}\right.$, 


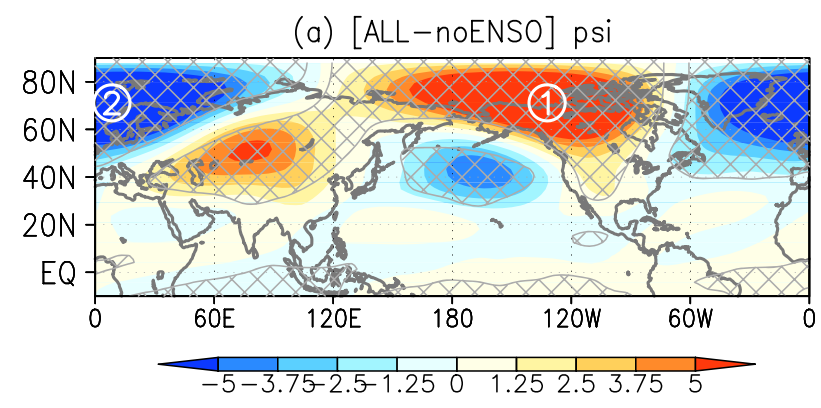

(b) $[\mathrm{ALL}-\mathrm{dtrSIC}] \mathrm{psi}$

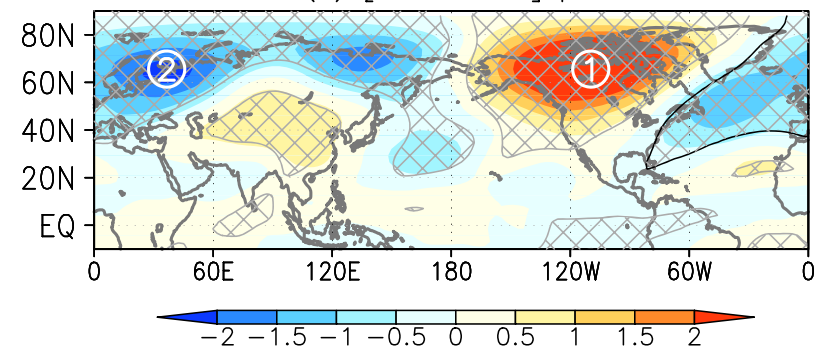

(c) PDF of psi amplitude (1-2)

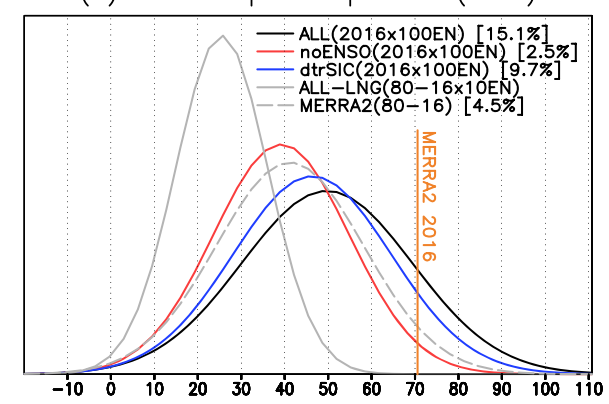

Fig. 4 The impacts of the El Nino and the sea-ice. The ensemble average responses of the stream function at $40 \mathrm{hPa}$ (shading; $10^{6} \mathrm{~m}^{2} \mathrm{~s}^{-1}$; zonal mean is removed) to (a) the El Nino (ALL minus noENSO) and (b) the sea-ice anomalies (ALL minus dtrSIC) in February 2016 based on the AGCM experiments of MIROC5. Hatchings denote a significance level of $90 \%$ based on the bootstrap method. c The normalized PDFs of the amplitudes of the extratropical disturbances for February 2016 in ALL (black line), noENSO (red line), and dtrSIC (blue line) experiments and for February 1980-2016 in MERRA2 (gray dashed line) and ALL-LNG (gray solid line). The amplitude is defined as the maximum value of stream function anomaly $\left(10^{6} \mathrm{~m}^{2} \mathrm{~s}^{-1}\right)$ over $\left(150^{\circ} \mathrm{E}-60^{\circ} \mathrm{W}, 50-80^{\circ} \mathrm{N}\right)$ minus the minimum value over $\left(60^{\circ} \mathrm{W}-150^{\circ} \mathrm{E}\right.$, $50-80^{\circ} \mathrm{N}$ ). Examples of the locations of the maxima and the minima are denoted as (1) and (2), respectively, in (a) and (b). The orange line indicates the amplitude of February 2016 in MERRA2. The bias corrections are applied to the PDFs of ALL, noENSO, and dtrSIC

$50-80^{\circ} \mathrm{N}$ ). Figure $4 \mathrm{C}$ shows the normalized probability distribution functions (PDFs) of the amplitudes, assuming a Gaussian distribution. A gray dashed line shows the PDF for 1980-2016 in MERRA2 (37 years $\times 1$ members). The PDFs for ALL, noENSO, and dtrSIC (1 year $\times 100$ members) are shown as black, red, and blue solid lines, respectively. We applied bias corrections to these PDFs (see Methods). The occurrence probabilities of amplitudes greater than those observed in 2016 are $15.1 \%$ in ALL, 2.5\% in noENSO, and $9.7 \%$ in dtrSIC. The probability is significantly reduced when the forcings of El Nino or the sea-ice anomaly are removed. The importance of El Nino and sea-ice is further illustrated by the calculated atmospheric responses to the heating associated with El Nino and the sea-ice, which were obtained using a linear atmospheric model (see supplementary).

\section{DISCUSIONS}

The stratospheric circulation structures of wavenumbers 1 and 2 are reminiscent of the leading mode of the tropospherestratosphere variation related to the Pacific/North American teleconnection pattern, ${ }^{15}$ which is often triggered by El Nino forcing. ${ }^{16-18}$ On the other hand, the sea-ice over the Barents and Kara Sea is suggested to influence the blocking high of the troposphere over Eurasia, ${ }^{9,10}$ and the associated tropospheric waves propagate upward into the stratosphere, resulting in the similar structures of wavenumbers 1 and $2 .^{18,19}$ In addition, the tropical lower stratosphere of early 2016 shows favorable conditions for wave propagation, with the westerly anomaly at approximately $20^{\circ} \mathrm{N}$. The extratropical disturbances being in close proximity to influence the QBO at $40 \mathrm{hPa}$.,6,8

The results of our analysis indicate that a tropical SST anomaly and Arctic sea-ice reduction can force the unusual disruption of the QBO observed in February 2016, which is shown using the multiple regression analyses using the observational/reanalysis data and is supported by the AGCM large-ensemble experiments and dynamic diagnosis by the linear model. The regression map of the lower stratospheric circulations shows the structures of wavenumbers 1 and 2 are similar to the horizontal distribution of the 2016 anomaly, accounting for about half of the 2016 anomaly. Moreover, the occurrence of the disruption may be sensitive to the QBO phase at the time of the extratropical wave forcing. As the winter begins with the westerly QBO and a strong EI Nino event/low sea-ice concentration, the extratropical disturbances act to decelerate the westerly winds and hence tends to disrupt the QBO, but not when the winters begins with the easterly QBO. Similar QBO disruptions may occur more frequently in the future warm climate due to the increasing stratospheric wave activities ${ }^{6}$ associated with the decreasing Arctic sea-ice ${ }^{20}$ and possible enhancement of the El Nino. ${ }^{21}$

\section{METHODS}

Data

This study used a monthly dataset of sea surface temperatures (SST) and sea-ice compiled by the Hadley Center (HadISST; https://www.metoffice. gov.uk/hadobs/hadisst).22 The atmospheric variables of the zonal wind, meridional wind, vertical pressure velocity, temperature, stream function (the zonal mean is removed to emphasize its zonal variations), and surface pressure were obtained from the daily datasets of the Modern-Era Retrospective analysis for Research and Applications, Version 2 (MERRA2; https://gmao.gsfc.nasa.gov/reanalysis/MERRA-2), ${ }^{23}$ the Japanese 55-year reanalysis (JRA55; http://jra.kishou.go.jp) ${ }^{24}$ and the European Center for Medium-Range Weather Forecasts Interim Re-Analysis (ERAl; https://www. ecmwf.int/en/forecasts/datasets/reanalysis-datasets/era-interim). ${ }^{25}$ The EP flux diagnosed using these datasets is scaled based on Edmon et al. ${ }^{26}$ The EP flux and WAF above $100 \mathrm{hPa}$ and equatorward of $30^{\circ} \mathrm{N}$ are multiplied by factors of 5 and 10 , respectively. The $40 \mathrm{hPa}$ level is available in MERRA2 but not in JRA55 or ERAI. The $40 \mathrm{hPa}$ in JRA55 and ERAI is interpolated using the 50 and $30 \mathrm{hPa}$ levels. The results using JRA55 and ERAl are similar to those using MERRA2 (Fig. S3). The analyzed period was 1980-2016. The climatology was defined as the 37-year average, and anomalies were deviations from the climatology. Although this study discusses analyses for the monthly averages of February, just before the unexpected easterly appears, similar relationships of the tropical wave convergence with $\mathrm{El}$ Nino and SIC are obtained for December to February (not shown). The statistical significances of the anomalies and differences were tested using a bootstrap method, in which the data samples were randomly shuffled 1000 times, and a significance level of $90 \%$ were denoted by hatchings in the figures. To account for autocorrelation in the original time series, we applied a moving block modification of the bootstrap technique. ${ }^{27}$

\section{AGCM}

The AGCM used in this study is the Model for Interdisciplinary Research on Climate, version 5 (MIROC5), ${ }^{28}$ which was jointly developed at the University of Tokyo, the National Institute for Environmental Studies, and the Japan Agency for Marine-Earth Science and Technology. The horizontal 
resolution of the model is T85 spectral truncation $(\sim 150 \mathrm{~km})$ and the model has 40 vertical levels of hybrid sigma-pressure vertical coordinates. We prescribed the observed monthly-mean SST and sea-ice concentrations in the model. SST and sea-ice at each time step are linearly interpolated from these monthly datasets. We used MIROC5 in the large-ensemble experiments as part of the attribution experiment. ${ }^{13}$ Although MIROC5 does not capture QBO variabilities because of its insufficient vertical resolution, it reasonably simulates responses of extratropical disturbances to SST and sea-ice, ${ }^{28}$ which are the focus of this study.

To evaluate the model climatology, we perform a control experiment for 1980-2016 (ALL-LNG; 10-member ensemble). Figure S4 shows the climatological average stream function of the lower stratosphere for MERRA2 (1980-2016 × 1 member) and ALL-LNG (1980-2016 × 10 member). The model captures the basic structure of the extratropical disturbances with a large negative value over northern Eurasia and a positive value around Alaska. Spectral analysis indicates that the dominant zonal wavenumbers of the disturbances are 1 and 2, both in MERRA2 and in the model. The ALL-LNG experiment simulates the horizontal distribution of the lower stratospheric circulations reasonably well. However, the model underestimates the amplitudes of the disturbances. To account for the model biases in calculating the PDFs in Fig. 4c, we define the bias corrected anomalies as

$$
\begin{aligned}
\text { Anomaly }_{\text {corrected }} & =\left(\text { Anomaly }_{\text {original }}-\mathrm{AVR}_{\mathrm{ALL}-\mathrm{LNG}}\right) \\
\times \frac{\text { STD }}{\text { MERRA2 }} & \mathrm{AVR}_{\text {MERRA2 }}
\end{aligned}
$$

where AVR and STD are the average and the standard deviations, respectively, for ALL-LNG and MERRA2. The PDF of ALL-LNG (a gray solid line in Fig. 4c) coincides with that of MERRA2 (a gray dashed line in Fig. 4c) after the corrections.

\section{Data availability}

All observational/reanalysis datasets are downloaded from their respective websites. The model data used in this study are available from the corresponding author on request.

\section{ACKNOWLEDGEMENTS}

This study was supported by the Integrated Research Program for Advancing Climate Models and KAKENHI $(15 \mathrm{H} 02132,15 \mathrm{KK} 0178,17 \mathrm{~K} 18816,26287117,26281013)$ of the Ministry of Education, Culture, Sports, Science, and Technology, Japan, and by the Environment Research and Technology Development Fund (2-1503; 2-1709) of the Environmental Restoration and Conservation Agency, Japan. The Earth Simulator at JAMSTEC and NEC SX-ACE at NIES were used to perform the model simulations. The Grid Analysis and Display System was used to plot the figures.

\section{AUTHOR CONTRIBUTIONS}

N.H., H.S., and H.A. designed the research. N.H. and H.S. performed the numerical experiments and analysis. N.H. wrote the paper. All authors discussed the results and commented on the manuscript.

\section{ADDITIONAL INFORMATION}

Supplementary information accompanies the paper on the npj Climate and Atmospheric Science website (https://doi.org/10.1038/s41612-018-0020-1).

Competing interests: The authors declare no competing financial interests.

Publisher's note: Springer Nature remains neutral with regard to jurisdictional claims in published maps and institutional affiliations.

\section{REFERENCES}

1. Baldwin, M. et al. The quasi-biennial oscillation. Rev. Geophys. 39, 179-229 (2001).

2. Naujokat, B. An update of the observed quasi-biennial oscillation of the stratospheric winds over the tropics. J. Atmos. Sci. 43, 1873-1877 (1986).

3. Kawatani, Y. \& Hamilton, K. Weakened stratospheric quasibiennial oscillation driven by increased tropical mean upwelling. Nature 497, 478-481 (2013).
4. Scaife, A. A. et al. Predictability of the quasi-biennial oscillation and its northern winter teleconnection on seasonal to decadal timescales. Geophys. Res. Lett. 41 1752-1758 (2014).

5. Newman, P., Coy, L., Pawson, S. \& Lait, L. The anomalous change in the QBO in 2015-2016. Geophys. Res. Lett. 43, 8791-8797 (2016).

6. Osprey, S. M. et al. An unexpected disruption of the atmospheric quasi-biennial oscillation. Science 353, 1424-1427 (2016).

7. Coy, L., Newman, P. A., Pawson, S., \& Lait, L. R. Dynamics of the disrupted 2015-16 quasi-biennial oscillation. J. Climate 30, 5661-5674 (2017).

8. Dunkerton, T. J. The quasi-biennial oscillation of 2015-2016: Hiccup or death spiral? Geophys. Res. Lett. 43 (2016).

9. Tang, Q., Zhang, X., Yang, X. \& Francis, J. A. Cold winter extremes in northern continents linked to Arctic sea ice loss. Environ. Res. Lett. 8, $014-036$ (2013).

10. Mori, M., Watanabe, M., Shiogama, H., Inoue, J. \& Kimoto, M. Robust arctic sea-ice influence on the frequent Eurasian cold winters in past decades. Nat. Geosci. 7 869-873 (2014).

11. Andrews, D. G. \& Mclntyre, M. E. Planetary waves in horizontal and vertical shear: the generalized Eliassen-Palm relation and the mean zonal acceleration. J. Atmos. Sci. 33, 2031-2048 (1976).

12. Takaya, K. \& Nakamura, H. A formulation of a phase independent wave-activity flux for stationary and migratory quasi-geostrophic eddies on a zonally varying basic flow. J. Atmos. Sci. 58, 608-627 (2001).

13. Shiogama, H. et al. An event attribution of the 2010 drought in the South Amazon region using the MIROC5 model. Atmos. Sci. Lett. 14, 170-175 (2013).

14. Christidis, N. \& Stott, P. A. Change in the odds of warm years and seasons due to anthropogenic influence on the climate. J. Clim. 27, 2607-2621 (2014).

15. Cheng, X. \& Dunkerton, T. J. Orthogonal rotation of spatial patterns derived from singular value decomposition analysis. J. Clim. 8, 2631-2643 (1995).

16. Baldwin, M. P. \& O'Sullivan, D. Stratospheric effects of ENSO-related tropospheric circulation anomalies. J. Clim. 8, 649-667 (1995).

17. Shiogama, H. \& Mukougawa, H. Influence of ENSO on the stratospheretroposphere coupling during stratospheric sudden warming events. Sola 1, 125-128 (2005).

18. Cheung, H. H. et al. A strong phase reversal of the Arctic Oscillation in midwinter 2015/2016: Role of the stratospheric polar vortex and tropospheric blocking. J. Geophys. Res. 121, 13443-13457 (2016).

19. Kodera, K., Mukougawa, H. \& Fujii, A. Influence of the vertical and zonal propagation of stratospheric planetary waves on tropospheric blockings. J. Geophys. Res. 118, 8333-8345 (2013).

20. Kim, B.-M. et al. Weakening of the stratospheric polar vortex by arctic sea-ice loss. Nat. Commun. 5, 4646 (2014).

21. Watanabe, M. et al., Uncertainty in the ENSO amplitude change from the past to the future. Geophys. Res. Lett. 39, L20703 (2012).

22. Rayner, N. et al. Global analyses of sea surface temperature, sea-ice, and night marine air temperature since the late nineteenth century. J. Geophys. Res. 108, 4407 (2003).

23. Bosilovich, M. G. et al. MERRA-2: Initial Evaluation of the Climate. Series on Global Modeling and Data Assimilation. NASA Technical Report, NASA/TM-2015-104606. Vol. 39, 136 (NASA, 2015).

24. Kobayashi, S. et al. The JRA-55 reanalysis: general specifications and basic characteristics. J. Meteorol. Soc. Jpn. 93, 5-48 (2015).

25. Dee, D. et al. The ERA-Interim reanalysis: configuration and performance of the data assimilation system. Quart. J. Roy. Meteor. Soc. 137, 553-597 (2011).

26. Edmon, H. Jr, Hoskins, B. \& McIntyre, M. Eliassen-Palm cross sections for the troposphere. J. Atmos. Sci. 37, 2600-2616 (1980).

27. Wilks, D. S. Resampling hypothesis tests for autocorrelated fields. J. Clim. 10, 65-82 (1997)

28. Watanabe, M. et al. Improved climate simulation by MIROC5: Mean states, variability, and climate sensitivity. J. Clim. 23, 6312-6335 (2010).

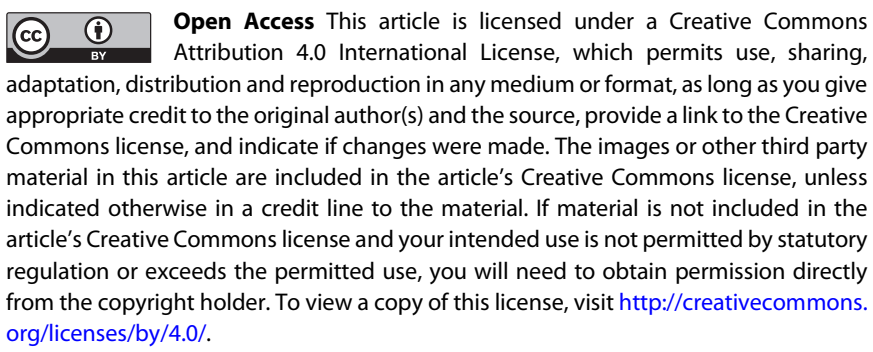

(c) The Author(s) 2018 